EPJ Web of Conferences 19, 06010 (2012)

DOI: $10.1051 /$ epjconf/20121906010

(C) Owned by the authors, published by EDP Sciences, 2012

\title{
Unifying the planar bar and the boxy bulge of the Milky Way
}

\author{
I. Martinez-Valpuesta ${ }^{\mathrm{a}}$ and O. Gerhard \\ Max-Planck-Institut für Extraterrestrische Physik, Giessenbachstrasse, \\ 85748 Garching, Germany
}

\begin{abstract}
For some time the Milky Way has been understood as a barred disk galaxy. Star count observations have provided evidence for two bars at apparently different orientations, the boxy bulge and a long planar bar. We report recent work in which we argued for a scenario where these observations can be reproduced with a single boxy bulge/bar: an evolved bar from the stellar disk and the corresponding boxy bulge generated from it through secular evolution and buckling instability. We calculated the star count distributions along different lines-of-sight for a simulated barred galaxy and an observer at the Sun position, and compared them with observations of red clump magnitude distributions. We found a good agreement between the model and the observations, even though the simulation has a single boxy bulge/bar. In this model, the different apparent orientations of the boxy bulge and planar bar are partially due to the volume effect and partially to the leading ends of the bar.
\end{abstract}

\section{INTRODUCTION}

During the last four decades it has become clear that our Galaxy is a barred system (see ref. [1]). From start counts, [2-4] found a boxy bulge of $\sim 2 \mathrm{kpc}$ length, with an orientation of $\alpha \sim 20^{\circ}-25^{\circ}$. When going toward larger longitudes, different authors [5-9] found signatures for a longer and flatter bar with an orientation around $\alpha \sim 43^{\circ}$. The two structures were detected at different longitudes: $|l|<12^{\circ}$, and generally above the plane for the boxy bulge; and $l$ between $20^{\circ}$ and $30^{\circ}$ for the flat long bar. These results are puzzling since such misaligned structures would be dynamically quite unstable. In [10] we proposed how both components could be understood as parts of a single boxy bulge-bar structure.

Such a bar consisting of an inner boxy bulge and a surrounding planar bar in the outer parts is a characteristic outcome of the secular evolution of barred galaxies [11], where the bar eventually buckles and forms a boxy-bulge, e.g., $[12,13]$. After the buckling event the bar resumes its evolution and continues to grow slowly through angular momentum exchange between bar, disk and dark matter halo [14-17]. In [10], we tested quantitatively that such a model predicts magnitude-dependent star count observations in approximate agreement with observations. These results are summarized below. General arguments for such a scenario have been independently given by [18] and by E. Athanassoula in her talk. For further tests some extra velocity predictions for general fields near the bar end are given.

\section{A MODEL FOR THE MILKY WAY'S BAR AND BULGE FORMED THROUGH SECULAR EVOLUTION}

The simulation we used in [10] is similar to that published in [17] and was not run to match the MW structure. The code used was FTM 4.4 (updated version) from [19]. The total number of particles is

\footnotetext{
a e-mail: imv@mpe.mpg.de
}

This is an Open Access article distributed under the terms of the Creative Commons Attribution-Noncommercial License 3.0, which permits unrestricted use, distribution, and reproduction in any noncommercial medium, provided the original work is properly cited. 

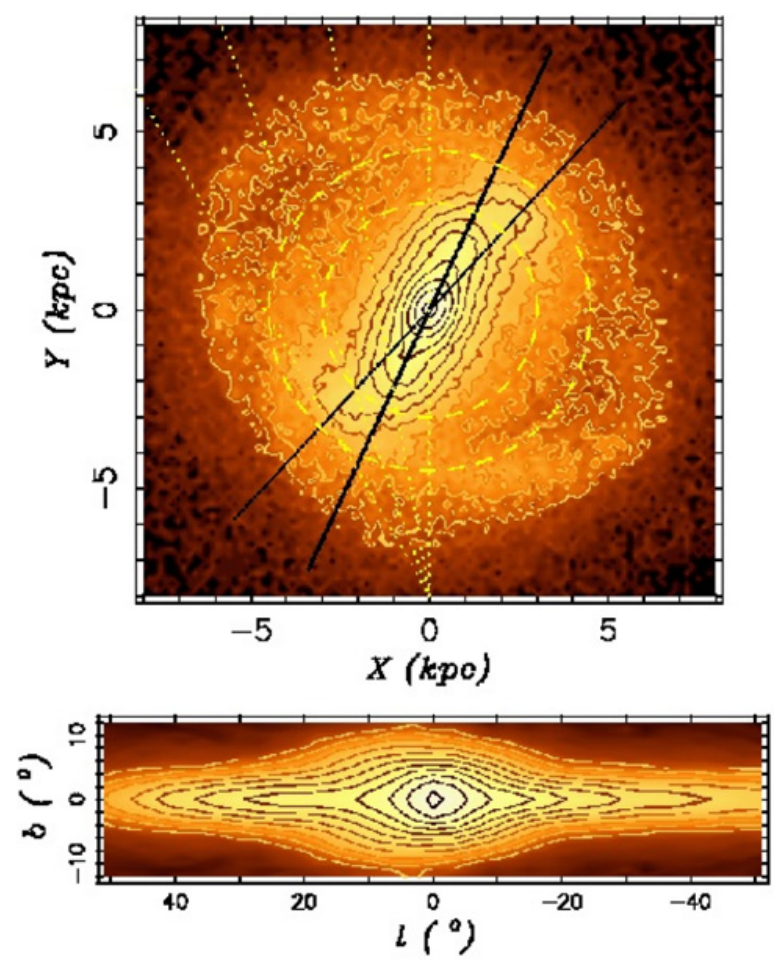

Figure 1. Left panel: Face-on view of the simulation at time $T \sim 1.9$ Gyr. The bar rotates clockwise. The model has been scaled to the MW and is oriented such that the long axis of the bulge is seen at an angle $\alpha=25^{\circ}$ by the observer at $(0,-8 \mathrm{kpc})$. A second line at $43^{\circ}$ as inferred for the long-bar in the MW is also shown. Right panel: edge-on view of the same snapshot, as viewed from the Sun. The boxy structure is noticeable. Higher densities correspond to brighter colours (as in [10]).

$1 \times 10^{6}$, distributed initially in an exponential disk with $Q=1.5$, embedded in a live dark matter halo. After $\sim 1.5 \mathrm{Gyr}$ the bar becomes very strong and buckles, thereby weakening. Later the bar resumes its evolution and grows again, resulting in a prominent boxy bulge and bar structure.

Figure 1 shows the simulated galaxy at time $\sim 1.9 \mathrm{Gyr}$, after the boxy bulge has formed and the bar has regrown. The face-on density distribution for this snapshot is shown in Figure 1a, oriented at an angle $\alpha=25^{\circ}$ with respect to the line from the Galactic centre to the observer. The boxy bulge is apparent in Figure $1 b$. The Sun was placed at $8 \mathrm{kpc}$. The model was scaled so that the end of the planar bar appears just inside longitude $l=30^{\circ}$ as seen from the observer. The bar length is $\sim 4.5 \mathrm{kpc}$, and the maximum ellipticity is 0.46 .

In the face-on view can be clearly seen the curved, leading ends of the stellar bar. Over time, the model shows oscillations from leading through straight to trailing ends and back. Similar morphology can be seen in other barred simulations in the literature (e.g. model m08, [22]; model B2.25, [23]) and also in some galaxies such as NGC 3124 and NGC 3450. The oscillations between trailing and leading ends of the bar could be related to the oscillations seen in the bar growth in N-body simulations (e.g. [24]) and may be due to non-linear coupling modes between the bar and spiral arms [25]. The leading ends of the bar are important for modelling the long bar observations.

\section{QUANTITATIVE ANALYSIS: NO NEED FOR A SEPARATE 'LONG BAR'}

In [10], we applied a similar technique as that used by observers to analyse the boxy bulge [2,3] and the long bar in the MW [6-9]. This technique consists in identifying the maxima of the magnitude 

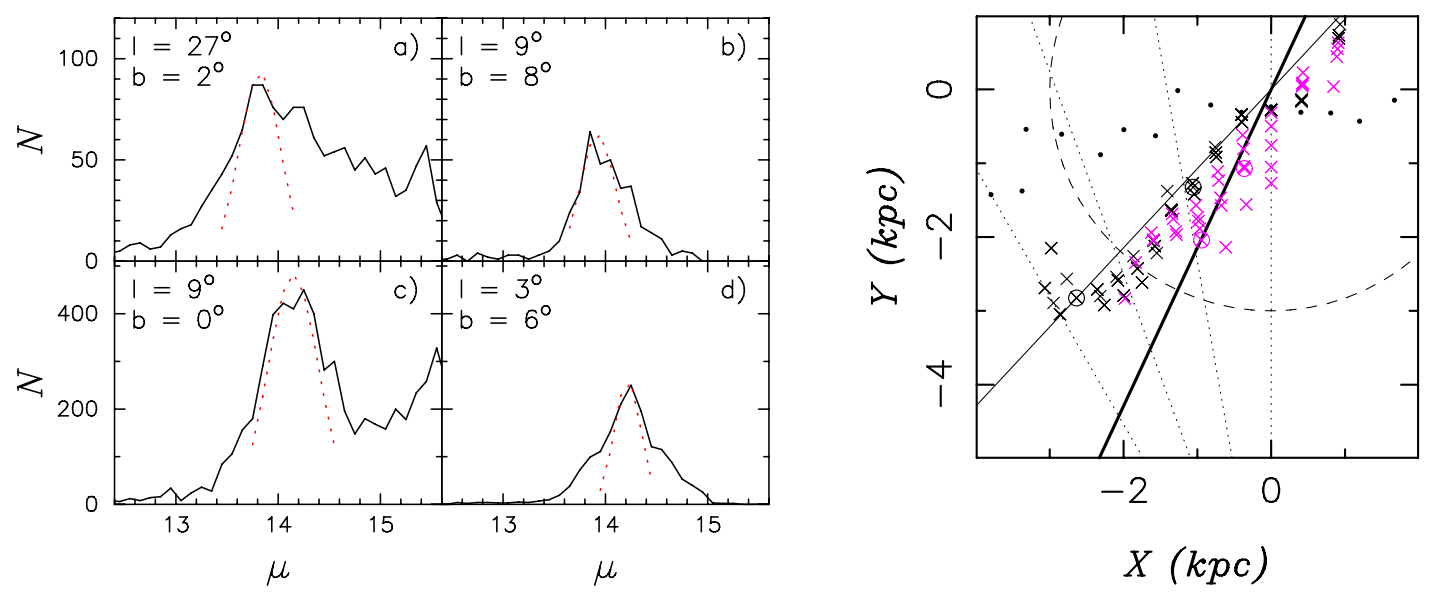

Figure 2. On the left, distribution of particles with distance modulus in four fields as seen by an observer at the Sun's position $8 \mathrm{kpc}$ from the centre (from [10]). The left panels $a$ ), $c$ ) show distributions in/near the Galactic plane in cones centred on the given longitude and latitude. The right panels $b$ ), $d$ ) show histograms for cones through the boxy bulge. On the right, maxima of particle distributions versus distance modulus, for all fields in the disk plane (black crosses) and in the boxy bulge $\left(4^{\circ} \leq|b| \leq 8^{\circ}\right.$, red crosses). The circled crosses correspond to the histograms shown on the left. The thick solid line shows the true orientation of the model, $\alpha=25^{\circ}$. The thin line follows $\alpha^{\prime}=43^{\circ}$. In order to increase the signal the simulation has been symmetrized vertically.

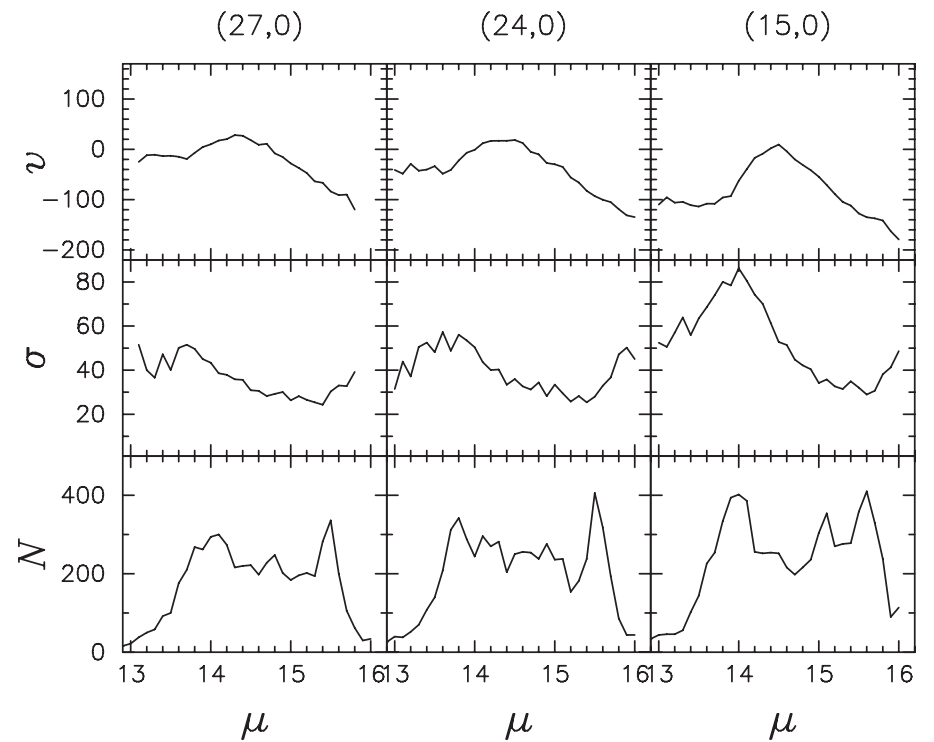

Figure 3. Dependence of mean radial velocity (top), velocity dispersion (middle), and particle number (bottom) on distance modulus, for three fields centred at $l=27^{\circ}, 24^{\circ}$ and at $15^{\circ}$ and $b=0^{\circ}$. The histograms show the bar signature as a lower radial velocity and higher velocity dispersion in the bar region (at $\mu \sim 14$ ).

distribution of red clump stars along various line-of-sight ( $(l, b)$ : correspond to longitude and latitude). Given the distribution of model particles with distance modulus (see Fig. 2 left) we fitted a Gaussian to the first peak. For comparison and clarification we show four histograms. Near the ends of the bar (see Fig. 2 left a), we can identify in the histogram three main peaks, one corresponding to the flat end 
of the bar, one to the spiral arm on the back and one corresponding to the end of the disk. The second histogram shows the distribution of stars when looking at a field well above the plane, but in the region of the thick structure (the boxy-bulge). The third histogram shows a field at the same longitude as the previous one but when looking through the plane. The fourth histogram shows a field close to the centre of the model. The maxima of the histograms together with those for other fields are projected on the MW plane in Fig. 2 right. We can see how there are two sets of points, those in the plane following the line of $\sim 43^{\circ}$ and the other group, following the line at $\sim 25^{\circ}$.

The maxima of the line-of-sight distance distributions in the Galactic plane occur at distances somewhat further than the maxima of the line-of-sight density distributions, due to the volume effect in the star counts. This effect is stronger in the galactic plane (Fig. 2 right). Assuming a plausible orientation $\left(\alpha=25^{\circ}\right)$, this explains part of the observational signature which was previously used to infer the existence of a second long bar. If in addition we choose a model snapshot where the bar has leading ends, as seen in Fig. 1, most of the long bar signature in the star count data can be reproduced. While not made specially to fit to the MW, this model thus illustrates that the traditional Galactic bar (the boxy bulge) and the more recently inferred long bar can plausibly be explained by a single boxy bulge/bar structure (more details in [10]).

\section{RADIAL VELOCITY PREDICTIONS FOR THE BAR REGION}

We determined the distributions of radial velocity (Fig. 3) with distance modulus for the model in Galactic plane fields near the inferred end of the planar bar in the model. We chose three different fields: two at the end of the bar, on the positive side, and one closer to the transition between the planar bar and the boxy bulge. These fields can be compared with upcoming radial velocity survey data for the inner MW in order to better constrain the structure of bar and disk near the end of the bar.

\section{References}

[1] Gerhard, O. 2002, ASPCS, Vol. 273, The Dynamics, Structure \& History of Galaxies, ed. Da Costa \& Jerjen, 73-+

[2] Stanek, K. Z., Udalski, A., Szymanski, M., et al. 1997, ApJ, 477, 163

[3] Babusiaux C., Gilmore G., Irwin M., 2005, MNRAS, 359, 985

[4] López-Corredoira, M., Cabrera-Lavers, A., \& Gerhard, O. E. 2005, A\&A, 439, 107

[5] Hammersley, P. L., Garzón, F., Mahoney, T. J., et al. 2000, MNRAS, 317, L45

[6] Benjamin, R. A., et al. 2005, ApJ, 630, L149

[7] Cabrera-Lavers, A., Hammersley, P. L., González-Fernández, C., et al. 2007, A\&A, 465, 825

[8] Cabrera-Lavers, A., González-Fernández, C., Garzón, F., et al. 2008, A\&A, 491, 781

[9] Churchwell, E., et al. 2009, PASP, 121, 213

[10] Martinez-Valpuesta I., Gerhard O., 2011, ApJ, 734, L20

[11] Athanassoula, E. 2005, MNRAS, 358, 1477

[12] Combes, F., Debbasch, F., Friedli, D., \& Pfenniger, D. 1990, A\&A, 233, 82

[13] Raha, N., Sellwood, J. A., James, R. A., \& Kahn, F. D. 1991, Nature, 352, 411

[14] Lynden-Bell, D., \& Kalnajs, A. J. 1972, MNRAS, 157, 1

[15] Athanassoula, E. 2003, MNRAS, 341, 1179

[16] Debattista, V. P., \& Sellwood, J. A. 2000, ApJ, 543, 704

[17] Martinez-Valpuesta, I., Shlosman, I., \& Heller, C. 2006, ApJ, 637, 214

[18] Romero-Gómez M., Athanassoula E., Antoja T., Figueras F., 2011, MNRAS, 1587

[19] Heller, C. H., \& Shlosman, I. 1994, ApJ, 424, 84 
Assembling the Puzzle of the Milky Way

[20] Bissantz, N., Englmaier, P., Binney, J., \& Gerhard, O. 1997, MNRAS, 289, 651

[21] Bissantz, N., \& Gerhard, O. 2002, MNRAS, 330, 591

[22] Fux, R. 1997, A\&A, 327, 983

[23] Rautiainen P., Salo H., 2000, A\&A, 362, 465

[24] Dubinski, J., Berentzen, I., \& Shlosman, I. 2009, ApJ, 697, 293

[25] Tagger, M., Sygnet, J. F., Athanassoula, E., \& Pellat, R. 1987, ApJ, 318, L43 